\title{
Bound states in a model of interaction of Dirac field with material plane
}

\author{
Yu. M. Pismak ${ }^{1, \star}$ and D. Yu. Shukhobodskaia ${ }^{1}$ \\ ${ }^{1}$ Saint-Petersburg State University
}

\begin{abstract}
In the framework of the Symanzik approach model of the interaction of the Dirac spinor field with the material plane in the $3+1$-dimensional space is constructed. The model contains eight real parameters characterizing the properties of the material plane. The general solution of the Euler-Lagrange equations of the model and dispersion equations for bound states are analyzed. It is shown that there is a choice of parameters of the model in which the connected states are characterized by dispersion law of a massless particle moving along the material plane with the dimensionless Fermi velocity not exceeding one.
\end{abstract}

\section{Introduction}

Recently, the physics of quantum macro-phenomena has attracted the attention of many researchers. For success of the theoretical investigations in this field it is important to build a model in which the most essential features of the mechanism of generating the studied quantum effect would be presented. If such model is constructed in the framework of usual quantum field theory[1], one needs a considerable modification of its methods, since the properties of the vacuum change essentially because of interaction of quantum fields fluctuations with macroscopic objects. In this way quantum macro-effects may appear in dynamics of material bodies which cannot be explained in by means of classical physics.

It was first shown theoretically in 1948 by Casimir [2]. In his model according to calculations carried out by him, the interaction of ideally conducting uncharged plates of planar capacitor with photon vacuum fluctuations generates an attraction force between ones. This phenomenon, called the Casimir effect (CE), is observed experimentally [3-6], and the emperical results obtained for materials with high conductivity are, with a high degree of accuracy, in agreement with theoretical ones.

At distances of 10 to $1000 \mathrm{~nm}$ the lows as classical and quantum physics are essential. They form a special nanophysics with its highly unusual phenomena, one of the striking example of which is the CE. Investigation of nanophysics is important not only from the general theoretical point of view, but there is every reason to hope for an effective practical use of their results in the creation of new technologies and devices.

The theoretical phenomena such as $\mathrm{CE}$ are studied very intensively in numerous works $[6,7]$. However often, these investigations are based on the simplified models which applicability area is very limited. This make it difficult to achieve a holistic understanding specifity of nanophysics and

^e-mail: ypismak@yahoo.com 
the internal relationship of its various manifestations. Therefore, they are not meaningless attempts to build and research models assigned for studying a wide range of nanophysical phenomena.

It is natural to assume that this type of models should be constructed on the basis of the most important, universal, well-established physical principles. From all the used currently methods, the closest to that is the Symansik approach [8] for modeling the interaction of quantum field system with classical macro object, which is presented by an additional action functional (defect action), concentrated in the spatial domain where, this inhomogeneity - a macroscopic object - is located. An important assumption is also that the standard requirements for quantum field models (locality, renormalizability, symmetry properties) are fulfilled.

The Symanzik approach has been used in many investigations and, in particular, for modeling the interaction of QED fields with two-dimensional objects [9-14].In this paper we use it for modeling the interaction of spinor Dirac field with material plane and studying the property of localized near one bound states.

\section{Statement of problem}

Within the Symanzik approach, the general form of the action functional modeling the interaction of the quantum field with material object (defect) can be written as:

$$
S(\varphi)=S_{V}(\varphi)+S_{d e f}(\varphi) .
$$

Here, $S_{V}$ is the action of the basic quantum field model, and $S_{d e f}$ is the defect action:

$$
S_{V}(\varphi)=\int L(\varphi(x)) d^{D} x, S_{d e f}(\varphi)=\int_{\Gamma} L_{d e f}(\varphi(x)) d^{D^{\prime}} x
$$

where $\Gamma$ is a subspace of dimension $D^{\prime} \leq D$ in a $\mathrm{D}$-dimensional space [8]. In the quantum electrodynamic (QED) the action $S_{V}(\varphi)$ is the functional of photon vector field $A_{\mu}(x)$ and Dirac spinor fields $\bar{\psi}(x), \psi(x)$,

$$
S_{V}(\varphi)=S(\bar{\psi}, \psi, A)=-\frac{1}{4} F_{\mu v} F^{\mu v}+\bar{\psi}(i \hat{\partial}-m+i e \hat{A}) \psi
$$

with $F_{\mu \nu}=\partial_{\mu} A_{v}-\partial_{v} A_{\mu}$. Gauge invariance, locality, and renormalizability as the basic principles of QED impose strong constraints on the possible form of the defect action $S_{d e f}(\varphi)$. Taking them into account by modeling the interaction of QED fields with a two-dimensional surface without charges and currents, one obtains the following result: $S_{d e f}(\varphi)=S_{d e f}(A)+S_{d e f}(\bar{\psi}, \psi)$. If the surface is defined by equation $\Phi(x)=0, x=\left(x_{0}, x_{1}, x_{2}, x_{3}\right)$. then $S_{d e f}(A)$ is the Chern-Simons action

$$
S_{d e f}(A)=\frac{a}{2} \int \varepsilon^{\lambda \mu \nu \rho} \partial_{\lambda} \Phi(x) A_{\mu}(x) F_{\nu \rho}(x) \delta(\Phi(x)) d^{4} x,
$$

where $\varepsilon^{\lambda \mu \nu \rho}$ is the totally antisymmetric tensor $\left(\varepsilon^{0123}=1\right)$, and the parameter $a$ is a dimensionless coupling constant. This expression is the most general form of a gauge-invariant functional of $A_{\mu}$ concentrated on a defect surface being invariant in respect to reparameterization of one and not having any negative-dimension parameters. The most general form of the defect action $S_{d e f}(\bar{\psi}, \psi)$ is written as

$$
S_{d e f}(\bar{\psi}, \psi)=\sum_{j=1}^{16} \int \alpha_{j} \bar{\psi}(x) \Gamma_{j} \psi(x) \delta(\Phi(x)) d^{4} x,
$$

where $\Gamma_{j}$ are the 16 basis Dirac matrices and $\alpha_{j}$ are dimensionless coupling constants. 
As the material defect we consider the plane $x_{3}=0$. In this case

$$
S(\bar{\psi}, \psi)=\int \bar{\psi}(x)\left(i \hat{\partial}-m+\Omega\left(x_{3}\right)\right) \psi(x) d x,
$$

and the matrix $\Omega\left(x_{3}\right)=Q \delta\left(x_{3}\right)$ describes the coupling of Dirac fields with the plane. Because $\Omega\left(x_{3}\right)$ and $\delta\left(x_{3}\right)$ have the dimension of mass, the matrix $Q$ is dimensionless. We suppose that the defect plane is isotropic and homogeneous. It means that $S(\bar{\psi}, \psi)$ must be be invariant under translation in $x_{0}-, x_{1}-, x_{2}-$ directions and rotation around $x_{3}$-axe. The most general form of the matrix $Q$ fulfilling this condition is

$$
Q=r_{1} \mathbf{1}+i r_{2} \gamma_{5}+r_{3} \gamma_{3}+r_{4} \gamma_{5} \gamma_{3}+r_{5} \gamma_{0}+r_{6} \gamma_{5} \gamma_{0}+i r_{7} \gamma_{0} \gamma_{3}+i r_{8} \gamma_{1} \gamma_{2}
$$

where 1 is the unit $4 x 4$ - matrix, $i=\sqrt{-1}, \gamma_{j}, j=0,1,2,3$ are the Dirac matrices and $\gamma_{5}=i \gamma_{0} \gamma_{1} \gamma_{2} \gamma_{3}$. For the $\gamma$-matrixes we use the following representation

$$
\begin{gathered}
\gamma_{0}=\left(\begin{array}{cccc}
1 & 0 & 0 & 0 \\
0 & 1 & 0 & 0 \\
0 & 0 & -1 & 0 \\
0 & 0 & 0 & -1
\end{array}\right), \gamma_{1}=\left(\begin{array}{cccc}
0 & 0 & 0 & 1 \\
0 & 0 & 1 & 0 \\
0 & -1 & 0 & 0 \\
-1 & 0 & 0 & 0
\end{array}\right), \\
\gamma_{2}=\left(\begin{array}{cccc}
0 & 0 & 0 & -i \\
0 & 0 & i & 0 \\
0 & i & 0 & 0 \\
-i & 0 & 0 & 0
\end{array}\right), \gamma_{3}=\left(\begin{array}{cccc}
0 & 0 & 1 & 0 \\
0 & 0 & 0 & -1 \\
-1 & 0 & 0 & 0 \\
0 & 1 & 0 & 0
\end{array}\right) .
\end{gathered}
$$

Taking the functional derivatives of action (1) with respect to $\bar{\psi}(x)$ and $\psi(x)$ we obtain the EulerLagrange equations :

$$
\left(i \hat{\partial}-m+\Omega\left(x_{3}\right)\right) \psi(x)=0,\left(\partial_{\mu} \bar{\psi}(x)\right) \gamma^{\mu}+\bar{\psi}(x)\left(m-\Omega\left(x_{3}\right)\right)=0 .
$$

It can be easily verified that $\bar{\psi}(x)=\psi^{*}(x) \gamma_{0}$, if $\gamma_{0} \Omega^{+}\left(x_{3}\right)=\Omega\left(x_{3}\right) \gamma_{0}$. This condition is fulfilled if parameters $r_{j}, 1 \leq j \leq 8$ are real. We supposed that it is the case.

Let us introduce the following notations

$$
\bar{x}=\left(x_{0}, x_{1}, x_{2}\right), \overline{p x}=p_{0} x_{0}-p_{1} x_{1}-p_{2} x_{2} .
$$

for parts of 4-component vectors and represent $\psi(x)=\psi\left(\bar{x}, x_{3}\right)$ as $\psi(x)=\psi_{s}(x)+\psi_{a}(x)$, where

$$
\psi_{s}(x)=\frac{1}{2}\left(\psi\left(\bar{x}, x_{3}\right)+\psi\left(\bar{x},-x_{3}\right)\right), \psi_{a}(x)=\frac{1}{2}\left(\psi\left(\bar{x}, x_{3}\right)-\psi\left(\bar{x},-x_{3}\right)\right) .
$$

Analysis of the possible singularities of spinor $\psi(x)$ at the plane $x_{3}=0$, made in [14], leads to the following: for $x_{3} \neq 0$, the field $\psi(x)$ satisfies the Dirac equation

$$
(i \hat{\partial}-m) \psi(x)=0,
$$

and the relation

$$
-i \gamma_{3} \psi_{a}(\bar{x})+Q \psi_{s}(\bar{x})=0
$$

holds at $x_{3}=0$.

It is shown in [14] that the general solution of (2) by $x_{3} \neq 0$ can be represented as

$$
\psi(x)=\frac{1}{(2 \pi)^{3}} \int e^{i \bar{p} \bar{x}} \psi_{+}\left(\bar{p}, x_{3}\right) d \bar{p}, \text { for } x>0, \psi(x)=\frac{1}{(2 \pi)^{3}} \int e^{i \bar{p} \bar{x}} \psi_{-}\left(\bar{p}, x_{3}\right) d \bar{p}, \text { for } x<0
$$


where

$$
\psi_{ \pm}\left(\bar{p}, x_{3}\right)=U\left(\bar{p}, x_{3}\right) \chi_{ \pm}(\bar{p}), U\left(\bar{p}, x_{3}\right)=e^{-i \gamma_{3}(\hat{\bar{p}}+m) x_{3}}=e^{-i \kappa(\bar{p}) x_{3}} P^{+}(\bar{p})+e^{i \kappa(\bar{p}) x_{3}} P^{-}(\bar{p}) .
$$

Here, $\chi_{ \pm}(\bar{p})$ are arbitrary spinors depending only on $\bar{p}$, and we used the notations

$$
\kappa(\bar{p})=\sqrt{\bar{p}^{2}-m^{2}}, P^{ \pm}(\bar{p})=\frac{1}{2}\left(\mathbf{1} \pm \frac{\gamma_{3}(\hat{\bar{p}}+m)}{\kappa(\bar{p})}\right) .
$$

It follows from (3) that $\chi_{+}(\bar{p}), \chi_{-}(\bar{p})$ satisfy the relation

$$
-i \gamma_{3}\left(\chi_{+}-\chi_{-}\right)+Q\left(\chi_{+}+\chi_{-}\right)=0
$$

which implies that

$$
\chi_{-}=S \chi_{+}, S=\left(i \gamma_{3}+Q\right)^{-1}\left(i \gamma_{3}-Q\right) .
$$

The matrices $P^{ \pm}(\bar{p})$ are projectors:

$$
P^{ \pm}(\bar{p}) P^{ \pm}(\bar{p})=P^{ \pm}(\bar{p}), P^{+}(\bar{p})+P^{-}(\bar{p})=1, P^{+}(\bar{p}) P^{-}(\bar{p})=P^{-}(\bar{p}) P^{+}(\bar{p})=0 .
$$

Each of them has two two-dimensional eigensubspaces $L^{+}$and $L^{-}$, which are represented as linear combinations of two mutually orthogonal eigenvectors $v_{1}^{ \pm}$and $v_{2}^{ \pm}$:

$$
\begin{array}{r}
L^{ \pm}\left(a_{1}^{ \pm}, a_{2}^{ \pm}\right)=a_{1}^{ \pm} v_{1}^{ \pm}+a_{2}^{ \pm} v_{2}^{ \pm}, \\
P^{ \pm}(\bar{p}) L^{ \pm}\left(a_{1}^{ \pm}, a_{2}^{ \pm}\right)=L^{ \pm}\left(a_{1}^{ \pm}, a_{2}^{ \pm}\right), P^{ \pm}(\bar{p}) L^{\mp}\left(a_{1}^{\mp}, a_{2}^{\mp}\right)=0 .
\end{array}
$$

Here, $a_{i}^{ \pm}, i=1,2$, are complex parameters, and $v_{i}^{ \pm}, i=1,2$, are spinors, which we choose as

$$
\begin{array}{r}
v_{1}^{+}=n_{1}^{+}\left(-h_{1}, h_{2}^{-}, 1, h_{3}^{-}\right), v_{2}^{+}=n_{2}^{+}\left(h_{1}, h_{2}^{+}, 1, h_{3}^{+}\right), \\
v_{1}^{-}=n_{1}^{-}\left(-h_{1}, h_{2}^{+}, 1,-h_{3}^{+}\right), v_{2}^{-}=n_{2}^{-}\left(h_{1}, h_{2}^{-}, 1,-h_{3}^{-}\right) \\
h_{1}=\frac{\sqrt{p_{0}^{2}-m^{2}}}{m+p_{0}}, h_{2}^{ \pm}=\frac{p_{0}^{2}-m^{2} \pm \sqrt{p_{0}^{2}-m^{2}} \kappa(\bar{p})}{\left(m+p_{0}\right)\left(p_{1}-i p_{2}\right)}, h_{3}^{ \pm}=\frac{\kappa(\bar{p}) \pm \sqrt{p_{0}^{2}-m^{2}}}{p_{1}-i p_{2}}
\end{array}
$$

with normalization constants $n_{1}^{ \pm}, n_{2}^{ \pm}$defined by conditions $\bar{v}_{1}^{ \pm} v_{1}^{ \pm}=\bar{v}_{2}^{ \pm} v_{2}^{ \pm}=1$. These spinors are eigenstates of the helicity operators

$$
\sigma(\vec{p})=\frac{i}{2|\vec{p}|}(\vec{p} \vec{s}), \vec{s}=\left(\gamma_{2} \gamma_{3},-\gamma_{1} \gamma_{3}, \gamma_{1} \gamma_{2}\right)
$$

They satisfy the relations

$$
\left.\sigma(\vec{p})\right|_{p_{3}=\mp \kappa(\bar{p})} v_{1}^{ \pm}=-\frac{1}{2} v_{1}^{ \pm},\left.\sigma(\vec{p})\right|_{p_{3}=\mp \kappa(\bar{p})} v_{2}^{ \pm}=\frac{1}{2} v_{2}^{ \pm}
$$

We represent the spinors $\chi_{ \pm}(\bar{p})$ as

$$
\chi_{ \pm}(\bar{p})=b_{ \pm}^{1}(\bar{p}) v_{1}^{-}(\bar{p})+b_{ \pm}^{2}(\bar{p}) v_{2}^{-}(\bar{p})+c_{ \pm}^{1}(\bar{p}) v_{1}^{+}(\bar{p})+c_{ \pm}^{2}(\bar{p}) v_{2}^{+}(\bar{p})
$$

If $\kappa>0$ the fields $\bar{\psi}, \psi$ describe the scattering processes of Dirac particles on the defect plane. For the model with $r_{5}=r_{6}=r_{7}=r_{8}=0$ they are studied in [14]. In this paper we consider the properties of bound states localized in vicinity of $x_{3}=0$. 


\section{Bound states}

The state is localized close to the plane $x_{3}=0$ if $\bar{p}^{2}-m^{2}<0$ and $\kappa(\bar{p})=i|\kappa(\bar{p})|$ is imaginary. In this case the spinors $\chi_{ \pm}(\bar{p})(5)$ must fulfill the conditions $b_{+}^{1}=b_{+}^{2}=c_{-}^{1}=c_{-}^{2}=0$. Other case the asymptotic of fields $\bar{\psi}(x), \psi(x)$ could be infinite by $x_{3} \rightarrow \pm \infty$. It follows from equation (4) that

$$
P^{-}(\bar{p}) S \chi_{+}=0
$$

It is the system of homogeneous linear equations for coefficients $b_{+}^{1}, b_{+}^{2}$. Its solvability condition has the form

$$
\left(p_{1}^{2}+p_{2}^{2}\right) R_{3}-\left(i \kappa R_{1}+2\left(m r_{18}^{-}-p_{0} r_{45}^{+}\right)\right)\left(i \kappa R_{2}+2\left(m r_{18}^{+}+p_{0} r_{45}^{-}\right)\right)=0
$$

where we used the notations $r_{j k}^{ \pm}=r_{j} \pm r_{k}$ and

$$
R_{1}=1+{r_{18}^{-2}}^{2}+r_{27}^{-2}+r_{36}^{+2}-r_{45}^{+2}, R_{2}=1+r_{18}^{+2}+r_{27}^{+2}+r_{36}^{-2}-r_{45}^{-2}, R_{3}=4\left(r_{6}^{2}+r_{7}^{2}+r_{8}^{2}-r_{4}^{2}\right) .
$$

The equation (7) is the dispersion relation for bound state.

The equation (4),(6) enable to express the spinor parameters $b_{-}^{1}, b_{-}^{2}, c_{+}^{1}, c_{+}^{2}$ in terms of one of them, which can be fixed by choosing normalization condition. The currents in bound state defined by spinors $\chi_{+}, \chi_{-}$are presented as

$$
J_{k}^{ \pm}\left(\bar{p}, x_{3}\right)=e^{-\left|\kappa x_{3}\right|} j_{k}^{ \pm}(\bar{p}), j_{k}(\bar{p})=\bar{\chi}_{ \pm}(\bar{p}) \gamma_{k} \chi_{ \pm}(\bar{p})
$$

with $j_{3}^{ \pm}=0$ and by $p_{0}>m$

$$
\begin{aligned}
& j_{1}^{+}=\frac{4 \omega\left(\left|b_{-}^{1}\right|^{2}+\left|b_{-}^{2}\right|^{2}\right) \omega p_{1}-\left(\left|b_{-}^{1}\right|^{2}-\left|b_{-}^{2}\right|^{2}\right)|\kappa| p_{2}}{\left(p_{0}+m\right)\left(\omega^{2}+|\kappa|^{2}\right)}, \\
& j_{2}^{+}=\frac{4 \omega\left(\left|b_{-}^{1}\right|^{2}+\left|b_{-}^{2}\right|^{2}\right) \omega p_{2}+\left(\left|b_{-}^{1}\right|^{2}-\left|b_{-}^{2}\right|^{2}\right)|\kappa| p_{1}}{\left(p_{0}+m\right)\left(\omega^{2}+|\kappa|^{2}\right)}, \\
& j_{1}^{-}=\frac{4 \omega\left(\left|c_{+}^{2}\right|^{2}+\left|c_{-}^{1}\right|^{2}\right) \omega p_{1}-\left(\left|c_{+}^{2}\right|^{2}-\left|c_{+}^{1}\right|^{2}\right)|\kappa| p_{2}}{\left(p_{0}+m\right)\left(\omega^{2}+|\kappa|^{2}\right)}, \\
& j_{2}^{-}=\frac{4 \omega\left(\left|c_{+}^{2}\right|^{2}+\left|c_{+}^{1}\right|^{2}\right) \omega p_{2}+\left(\left|c_{+}^{2}\right|^{2}-\left|c_{+}^{1}\right|^{2}\right)|\kappa| p_{1}}{\left(p_{0}+m\right)\left(\omega^{2}+|\kappa|^{2}\right)},
\end{aligned}
$$

where $\omega=\sqrt{p_{0}^{2}-m^{2}}$. We see that the currents flow along the plane $x_{3}=0$ and are parallel to the momentum $\vec{p}=\left(p_{1}, p_{2}\right)$ if $b_{-}^{1}|=| b_{-}^{2}|,| c_{+}^{2}|=| c_{+}^{1} \mid$. The currents above and belove of the plane $x_{3}=0$ are parallel if $b_{-}^{1}|/| b_{-}^{2}|=| c_{+}^{2}|/| c_{+}^{1} \mid$.

If by a special choosing the parameters in the model $R_{1}=R_{2}=r_{18}^{+}=r_{18}^{-}=0$, then the dispersion relation (7) has the form

$$
p_{0}^{2}-v_{F}^{2}\left(p_{1}^{2}+p_{2}^{2}\right)=0
$$

where $V_{F}$ is a constant, which is expressed in the term of parameters $r_{k}, 1 \leq k \leq 8$ of the model. The dispersion low (8) describes the movement of massless particle with Fermi-velocity $V_{f}$. The motion of such particles explains numerous effects in graphene.

The constants $R_{1}, R_{2}, r_{18}^{+}, r_{18}^{-}$are equal to zero by $r_{1}=r_{8}=r_{3}=0$ and $r_{45}^{+}=$ $\pm \sqrt{1+r_{27}^{-2}+r_{6}^{2}}, r_{45}^{-}= \pm \sqrt{1+r_{27}^{+2}+r_{6}^{2}}$. For such choosing of parameters the Fermi-velocity $V_{F}$ 
can obtain two different values: $V_{F}=V_{F}^{+}$or $V_{F}=V_{F}^{-}$,

$$
V_{F}^{ \pm}=\sqrt{\frac{1}{2}\left(1 \pm \frac{1+r_{27}^{+} r_{27}^{-}-r_{6}^{2}}{\sqrt{1+r_{27}^{-2}+r_{6}^{2}} \sqrt{1+r_{27}^{+2}+r_{6}^{2}}}\right)}
$$

The Fermi-velocity $V_{F}^{+}\left(V_{F}^{-}\right)$corresponds to the positive (negative) value of $r_{45}^{+} r_{45}^{-}$. By changing the values of parameters $r_{2}, r_{6}, r_{7}$ one can obtain each value of the Fermi-velocity in the interval $(0,1)$. The current flowing along the plane $x_{3}=0$ is parallel to the momentum $\vec{p}$ only by $r_{6}=0$.

\section{Conclusions}

In the framework of the Symanzik approach, we built the most general model of spinor field interacting with the homogeneous isotropic material plane. The action of the model includes both the usual spinor Dirac action and additional defect contribution. It contains 8 real dimensionless parameters that characterize the property of material of defect. The Euler-Lagrange equations of the model describe both the scattering of Dirac particles on the plane and the bound states localized in its vicinity. The dispersion relation for the bound states is considered. It was shown that by a special choose of the model parameter it describe the motion of massless particle with Fermi-velocity $0<V_{F}<1$. The currents generated by particles moving along the material plane are parallel to the moment only by special values of the defect action parameters. The presented approach could be used for the theoretical description of the interaction of electrons, positrons and neutrons with two-dimensional materials (graphene, thin films, sputters, sharp boundaries of a solid body). It could be also fruitful for modeling of wide class system characterizing by two-dimensional quantum macro effects (the Hall and plasmon-polariton effects, topological insulators, nanophotonics [15] ). Simple modifications of the model allows to take into account the effects of external electromagnet ic fields.

We have constructed a model of the interaction of a spinor field with a material plane in the framework of Symanzik's approach. The action functional of the model includes the standard spinor Dirac action in $(3+1)$ dimensional space-time and an additional contribution from the defect, concentrated in the part of space that it occupies (in considered case it was the plane $x_{3}=0$ ). The action contains three parameters characterizing the properties of the material of the plane. In the model, we calculated the characteristics of a Dirac particle scattering on the defect plane and also studied the properties of states localized near it.

The model and the obtained results can be used to describe theoretically processes of the interaction of electrons, with two-dimensional materials (graphene, thin films, sputter, sharp boundaries of a solid body). Simple modifications of the model allow taking the effects of the action of external electromagnetic fields into account.

Applying Symanzik's approach to QED enables a construction of a model for a wide class of quantum macroscopic phenomena in two-dimensional materials. These models can prove usefulness in investigating the Hall effect, plasmon-polariton effects, nanophotonics, properties of topological insulators, thin films, sputters, and sharp boundaries [15].

Acknowledgments. This paper was supported in part by RFBR grant 16-02- 00943-a.

\section{References}

[1] N. N. Bogoliubov and D. V. Shirkov, Quantum Fields, Nauka, Moscow (1993); C. Itzykson and J.-B. Zuber Quantum Fields Theory, McGraw-Hill, New York (1980). 
[2] H. B. G Casimir, Proc. K. Ned. Akad. Wet B. 51, 793-795 (1948).

[3] U. Mohideen and A. Roy, Phys. Rev. Lett. 81, 4549-4552 (1998); arXiv:physics/9805038v2 (1998);

A. Roy, C.-Y. Lin and U. Mohideen, Phys. Rev. D 60, 111101(R) (1999); arXiv:quantph/9906062v3 (1999).

[4] B. W. Harris, F. Chen and U. Mohideen, Phys. Rev. A 62, 052109 (2000); arXiv:quantph/0005088v1 (2000).

[5] G. Bressi, G. Carugno, R. Onofrio and G. Ruoso, Phys. Rev. Lett. 88, 041804 (2002); arXiv:quant-ph/ 0203002v1 (2002).

[6] M. Bordag, G. L. Klimchitskaya, U. Mohideen, and V. M. Mostepanenko Advances in the Casimir Effect, (Intl. Ser. Monogr. Phys., Vol. 145), Oxford Univ. Press, Oxford (2009); G. L. Klimchitskaya, U. Mohideen and V. M. Mostepanenko, Rev. Mod. Phys. 81, 1827-1885 (2009); arXiv:0902.4022v1 [cond-mat.other] (2009).

[7] K. A. Milton, J. Phys. A: Math. Gen. 37, R209-R277 (2004).

[8] K. Symanzik, Nucl.Phys. B 190, 1-44 (1981). .

[9] V. N. Markov and Yu. M. Pis'mak, J. Phys. A: Math. Gen. 39, 6525-6532 (2006); arXiv: hepth/0505218v3 (2005).

[10] V. N. Marachevsky and Yu. M. Pis'mak, Phys. Rev. D 81, 065005 (2010); arXiv:0907.1985v2 [hep-th] (2009).

[11] D.Yu. Pis'mak and Yu. M. Pis'mak, Theor. Math. Phys. 175,816-826 (2013); Phys. Part. Nuclei 44, 450-461 (2013); Theor. Math. Phys. 166, 1423-1431 (2011).

[12] D.Yu. Pis'mak, Yu. M. Pis'mak and F. J. Wegner, Phys. Rev. E 92, 013204 (2015); arXiv:1406.1598v1 [hep-th] (2014).

[13] I. V. Fialkovsky, V. N. Markov and Yu. M. Pis'mak, J. Phys. A: Math. Gen. 39, 6357-6363 (2006); Int. J. Mod. Phys. A 21, 2601-2616 (2006); arXiv:hep-th/0311236v2 (2003).

[14] D. Yu. Pismak, Yu. M. Pismak, Theor. Math. Phys. 184, 3, 1329-1341 (2015).

[15] G. Bracco and B. Holst (Eds.), Surface Science Techniques, (Springer Ser. Surf. Sci., Vol. 51), Springer, Berlin (2013); M. L. Brongersma and P. G. Kik (Eds.), Surface Plasmon Nanophotonics, (Springer Ser. Opt. Sci., Vol. 131), Springer, Berlin (2007); S. A. Maier Plasmonics: Fundamental and Applications, Springer, Berlin (2007). 